\title{
Criminologie
}

\section{Technologies, déqualification et luttes d'influence chez les professionnels de la sécurité frontalière}

\section{Karine Côté-Boucher}

Volume 47, numéro 2, automne 2014

Criminalité et police transnationales : une perspective critique

URI : https://id.erudit.org/iderudit/1026731ar

DOI : https://doi.org/10.7202/1026731ar

Aller au sommaire du numéro

\section{Éditeur(s)}

Les Presses de l’Université de Montréal

ISSN

0316-0041 (imprimé)

1492-1367 (numérique)

Découvrir la revue

Citer cet article

Côté-Boucher, K. (2014). Technologies, déqualification et luttes d'influence chez les professionnels de la sécurité frontalière. Criminologie, 47(2), 127-151.

https://doi.org/10.7202/1026731ar
Résumé de l'article

Les travaux sur la sécurité frontalière ont clairement établi que les technologies se situent au coeur de la reconstitution des activités de police frontalière et de l'espace occupé par les frontières. Cet article a pour but d'ouvrir ces perspectives vers le monde peu étudié des douanes, c'est-à-dire là où l'on se préoccupe de la traçabilité des marchandises et des déplacements des travailleurs du transport. En nous appuyant sur une recherche de terrain menée dans les divisions " commerciales " de cinq postes frontaliers canadiens, nous posons la question des effets concrets de l'intégration des technologies dans les procès de travail douaniers. Nous nous penchons sur la manière dont les douaniers se constituent comme acteurs de sécurité, mais également sur les pratiques quotidiennes des professionnels de la sécurité frontalière, les conditions de travail et les relations intraorganisationnelles au sein de ces agences de sécurité frontalières. En effet, plutôt qu'une efficience accrue qui serait obtenue par le recours aux technologies, cette intégration présente des écueils qui révèlent, en creux, des luttes d'influence entre acteurs de terrain et cadres intermédiaires avec, en trame de fond, la déqualification et l'automatisation du travail douanier. 


\title{
Technologies, déqualification et luttes d'influence chez les professionnels de la sécurité frontalière
}

\author{
Karine Côté-Boucher ${ }^{1}$ \\ Professeure adjointe \\ École de criminologie, Université de Montréal \\ karine.cote-boucher@umontreal.ca
}

RÉSUMÉ - Les travaux sur la sécurité frontalière ont clairement établi que les technologies se situent au cœur de la reconstitution des activités de police frontalière et de l'espace occupé par les frontières. Cet article a pour but d'ouvrir ces perspectives vers le monde peu étudié des douanes, c'est-à-dire là où l'on se préoccupe de la traçabilité des marchandises et des déplacements des travailleurs du transport. En nous appuyant sur une recherche de terrain menée dans les divisions "commerciales» de cinq postes frontaliers canadiens, nous posons la question des effets concrets de l'intégration des technologies dans les procès de travail douaniers. Nous nous penchons sur la manière dont les douaniers se constituent comme acteurs de sécurité, mais également sur les pratiques quotidiennes des professionnels de la sécurité frontalière, les conditions de travail et les relations intraorganisationnelles au sein de ces agences de sécurité frontalières. En effet, plutôt qu'une efficience accrue qui serait obtenue par le recours aux technologies, cette intégration présente des écueils qui révèlent, en creux, des luttes d'influence entre acteurs de terrain et cadres intermédiaires avec, en trame de fond, la déqualification et l'automatisation du travail douanier.

мотS-CLÉs - Sécurité frontalière, douanes, technologies, déqualification, luttes d'influence.

\section{Introduction}

Les travaux sur la sécurité frontalière ont clairement établi que les technologies se situent au cœur de la reconstitution des frontières et de

1. Université de Montréal, École de criminologie, Pavillon Lionel-Groulx, C. P. 6128, succursale Centre-ville, Montréal (Québec), Canada, H3C 3J7. 
l'espace qu'elles occupent, participant non seulement d'une logique d'identification des personnes, mais répondant également à une rationalité plus générale qui promeut la surveillance des circulations et l'interception sélective des voyageurs et des migrants selon une logique préventive. Bien que ces tendances soient avérées (Amoore, 2006; Broaders, 2007; Pickering \& Weber, 2006; Scherrer et al. 2010), on s'est peu intéressé au rôle joué par les technologies dans les activités de traçabilité des marchandises et de surveillance des déplacements des travailleurs du transport, c'est-à-dire les douanes. Or, si les flux de migrants et de voyageurs sont souvent synonymes de circulation dans les études portant sur les frontières, la facilitation des mobilités de marchandises légales représente un aspect fondamental du contrôle frontalier contemporain. Ces opérations reposent sur une infrastructure technologique substantielle et en transformation dont il s'agit de mieux cerner les effets sur le travail frontalier.

Nous proposons de déplacer cette réflexion depuis son terrain de prédilection, à savoir les logiques qui sous-tendent la sécurisation contemporaine des frontières au moyen de technologies de l'information et de surveillance, vers l'étude de leurs usages concrets dans la gouvernance des frontières. Nous nous concentrons ainsi sur la pratique de la sécurité frontalière (Côté-Boucher, Infantino \& Salter, 2014) et sur les dynamiques de mise en œuvre des politiques en matière frontalière. À ce titre, nous souhaitons poser la question des conséquences de l'intégration quotidienne des technologies de l'information dans les procès de travail des douaniers. Pour ce faire, nous n'interrogeons pas l'outil technique en soi. Nous prenons plutôt cet outil du point de vue de son utilisation par des professionnels de la sécurité en examinant comment il travaille les organisations policières de l'intérieur. Il s'agit donc de prêter attention aux façons dont la technique s'intègre dans les pratiques quotidiennes des professionnels de la sécurité ainsi qu'à son influence sur leurs conditions de travail et sur les relations intraorganisationnelles dans les agences de sécurité.

Nous proposons donc de discuter de ces enjeux en nous appuyant sur un terrain d'enquête effectué dans les sections «commerciales» (là où l'on assure la mainlevée pour les marchandises, les camions et les camionneurs) de cinq ports d'entrée situés à la frontière avec les ÉtatsUnis et placés sous la responsabilité de l'Agence des services frontaliers $\mathrm{du}$ Canada (ASFC). Nous nous référerons plus particulièrement à 32 entrevues obtenues avec des travailleurs de première ligne rencontrés 
dans ces postes - incluant un analyste de renseignement, un chef des opérations (le gestionnaire principal d'un port d'entrée majeur ou d'une série de petits et moyens ports), deux adjointes administratives, cinq superviseurs et 23 agents des services frontaliers (connus auparavant sous le nom de «douaniers»).

Cet article se penche sur la construction d'un nouveau «régime douanier» (Chalfin, 2007) depuis la fin des années 1990 à travers l'adoption de la transmission électronique de la documentation sur le fret, les possibilités d'analyse de risque et de conformité douanière que cette informatisation a générées ainsi que l'intégration récente de la gestion des risques et de la relâche douanière. On note donc la multiplication d'interventions technologiques qui visent à faciliter et à réguler les flux de marchandises au moyen de ce que je propose d'appeler la «douane à distance». Ce recours aux technologies a profondément modifié les dynamiques spatio-temporelles constitutives des routines de travail douanières tout en renouvelant les enjeux de pouvoirs qui se jouent au sein des agences de sécurité. À ce titre, une automatisation et une redistribution des tâches au sein, mais également entre les postes frontaliers, ont partiellement déqualifié les douaniers. Les autorités frontalières ont également retiré aux agents de première ligne plusieurs responsabilités tout en déplaçant une partie significative de la décision douanière en amont du port d'entrée. Par conséquent, nous suggérons que la douane à distance tend à diminuer l'effectivité concrète des pouvoirs discrétionnaires formels des douaniers, des pouvoirs historiquement situés au cœur de l'identité occupationnelle des officiers frontaliers de terrain. Non seulement cette remise en question occasionne-t-elle une adoption ambivalente des nouveaux moyens techniques par les douaniers, mais elle se voit reflétée dans des tensions entre ces travailleurs de la sécurité et les gestionnaires en poste à la capitale. Ainsi, plutôt que de témoigner d'une efficacité accrue qui serait obtenue par le recours aux technologies, l'incorporation de ces dernières dans les routines de travail douanières présente des écueils qui révèlent, en creux, des rivalités internes entre acteurs de terrain et cadres intermédiaires avec, en trame de fond, la déqualification et l'automatisation du travail douanier. Par conséquent, en s'intéressant à la conduite quotidienne du contrôle des marchandises dans les espaces frontaliers, il est possible de rediriger les travaux sur les frontières vers l'étude des dimensions concrètes du recours aux technologies et celle des luttes 
d'influence que ce recours suscite au sein des organisations de sécurité frontalière contemporaines.

\section{Vers une sociologie des enjeux politiques et pratiques des technologies de sécurité}

Le travail d'identification, de fouille et d'examen a historiquement constitué l'essentiel du métier de douanier. Développées par les États modernes afin d'établir leur souveraineté territoriale à travers une «monopolisation des moyens légitimes de circulation» (Torpey, 2000), le long des frontières géopolitiques des États, ces activités continuent à être exercées quotidiennement dans les ports d'entrée à travers le monde. Elles supposent l'établissement d'une dynamique de pouvoir distinctive entre ce corps policier (désigné, selon les pays, sous le vocable de sécurité frontalière, douane, patrouille frontalière ou police des frontières) et ceux dont il régule les déplacements. Le travail frontalier est soutenu par un pouvoir discrétionnaire sans commune mesure dans la communauté policière - notamment caractérisé par la capacité de questionner et de détenir les individus ainsi que d'inspecter véhicules, bagages et marchandises sans autorisation judiciaire préalable. Mais au-delà des discussions sur le statut juridique et politique de l'autorité des polices frontalières et transnationales (Doty, 2007; Huysmans, 2008; Salter, 2008), ces pouvoirs reposent sur un acquis expérientiel constitué, transmis et réinterprété au sein des organisations sécuritaires. Souvent qualifiés d'intuition professionnelle et de «sixième sens», ces savoirs favorisent la mise en pratique d'une logique de suspicion chez ces acteurs de la sécurité frontalière (Gilboy, 1991; Heyman, 1995 ; Pratt \& Thompson, 2008), en même temps que cette suspicion s'autorise de représentations «quasi scientifiques» du risque (Pratt, 2010).

Cependant, la sécurité s'appuie de plus en plus sur diverses technologies d'identification, d'évaluation et de contrôle des mobilités, ce qui entraîne des conséquences importantes non seulement pour notre représentation des frontières, mais également pour la pratique de la sécurité frontalière. D'une part, la "technologisation» des frontières (Ceyhan, 2008) remet en question les conceptions traditionnelles de l'espace frontalier. Une littérature multidisciplinaire substantielle théorise la sécurité des frontières en tant qu'ensemble de techniques et de rationalités qui inaugurent la frontière contemporaine comme espace 
élargi de contrôle des circulations (cf., entre autres, Balibar, 2009; Squire, 2011; Weber \& Bowling, 2004). L'introduction de technologies de l'information et de surveillance dans la sécurisation des frontières s'inscrit donc dans une logique de gouvernance de la mobilité qui permet de redistribuer l'espace frontalier au-delà et au sein des territoires qu'elles sécurisent. La catégorisation de ces mobilités à des fins de traitement différentiel est inscrite dans ces moyens de contrôle. Lorsqu'elle est appliquée à des espaces frontaliers, la gestion des risques - cette stratégie de «triage social» (Lyon, 2007) - permet la classification des mobilités qui doivent être privilégiées et celles qui peuvent être entravées et même proscrites (Aas, 2011 ; Rygiel, 2010).

Dans cette optique, nous avons examiné ailleurs la manière dont la frontière canado-américaine a été spatialement diffusée en tant que «frontière intelligente» (smart border) (Côté-Boucher, 2008). Le caractère «intelligent» de la frontière s'exprime dans le recours à des technologies de l'information et à des moyens d'identification centrés sur le corps (par exemple biométrie et scanneurs corporels) que discours et politiques sécuritaires présentent comme gages d'une action de sécurité efficiente. D'autres travaux ont montré que cette entreprise de contrôle des mobilités, qui déploie les frontières au-delà de leur emplacement géopolitique au moyen de technologies de l'information et de recours à l'outil biométrique, n'est pas particulière à l'Amérique du Nord. Elle caractérise également les pratiques frontalières des autorités européennes et australiennes (Jeandesboz, 2010; Wilson \& Weber, 2008).

Cette diffusion des frontières fait émerger une «nouvelle topographie du contrôle frontalier» (Walters, 2006) que soutient une «pixellisation» des frontières (Bonditti, 2005) centrée sur la surveillance des flux au moyen d'ensembles de points de contrôle plus ou moins interconnectés. Elle repose également sur ce qui est maintenant convenu de nommer «une police à distance» (Bigo \& Guild, 2005) des individus et des mobilités basée sur la collecte, l'analyse et l'échange de données et renseignements entre instances policières, services de renseignement et agences frontalières. Dans ces conditions, la sécurité frontalière ne peut être regardée comme une activité neutre, mais comme un ensemble de pratiques dessinant un espace de pouvoir dont le centre décisionnel est déplacé en amont avec des effets significatifs sur l'imputabilité des processus sécuritaires. Amoore et de Goede (2008, p. 8) affirment ainsi qu'au sein de ces processus, "[the] decisions concerning which passengers need "a closer look" and which qualify for "faster service" are 
displaced inside bureaucratic and technological spaces that are difficult to understand, and even more difficult to challenge ${ }^{2}$ » (cf. également Aas, 2006).

D'autre part, si les considérations des études critiques sur les frontières permettent de réfléchir aux logiques sous-jacentes qui soutiennent un recours croissant aux technologies de l'information et de surveillance, elles évitent néanmoins la question de l'intégration concrète de ces moyens techniques de contrôle dans les pratiques quotidiennes des professionnels de la sécurité. Prêter attention à ces pratiques permet de déplacer le regard au-delà des dynamiques de classification particulières aux espaces frontaliers, et de s'intéresser aux acteurs sécuritaires euxmêmes, à leurs motivations diverses, aux contradictions quotidiennes auxquelles ils font face ainsi qu'aux manières plurielles dont ils font valoir leurs intérêts. En effet, le contrôle frontalier s'appuie sur les «savoirs pratiques locaux» (Infantino \& Rea, 2012; voir également Heyman, 2004) d'une série d'intervenants dotés de cultures occupationnelles distinctes et travaillant dans des environnements organisationnels particuliers - les aéroports, les bureaux de renseignement, les ports d'entrée terrestres, les entrepôts douaniers, les centres d'analyse de risque, les autorités d'immigration, les ambassades, etc. Ces acteurs de sécurité peuvent entrer dans des relations de collaboration et d'entraide, mais peuvent également entretenir des antagonismes et des rivalités; les technologies s'inscrivent donc dans cette pluralité de relations. On peut donc poser la question: quelles sont les conséquences de la diffusion technologique des frontières sur la pratique professionnelle des agents frontaliers de première ligne?

On peut trouver du côté de la sociologie du policing des recherches susceptibles d'éclairer empiriquement les interactions entre professionnels de la sécurité et technologies. Si le travail policier diffère sensiblement du travail de sécurité frontalière (notamment en ce qui a trait au pouvoir discrétionnaire), ces travaux proposent des pistes analytiques intéressantes pour la considération de ces interactions. En fait, si les bases de données et les activités de collecte et d'analyse qu'elles autorisent apparaissent intrinsèquement liées au changement organisationnel et occupationnel dans la pratique policière (Chan, Brereton, Legosz \& Doran, 2001 ; Ericson \& Haggerty, 1997), elles le sont tout autant dans le champ de la sécurité frontalière. Puisqu'elles produisent une

2. Les auteures soulignent. 
nouvelle matérialité de l'action de sécurité, ces technologies se retrouvent donc au cœur de transformations dans les façons d'agir, de penser et de sentir des travailleurs de la sécurité (Chan, 2001).

$\mathrm{Si}$, trahissant une assurance encore répandue dans des capacités qui seraient intrinsèques à l'outil technique, les technologies sont souvent appréhendées comme offrant des solutions tangibles et immédiates à divers problèmes de sécurité, il s'agit ici de s'intéresser aux défis posés et aux tensions créées par leur intégration concrète dans la pratique sécuritaire. D'abord, les technologies modifient les routines de travail sécuritaires. En effet, les activités policières sont maintenant organisées et évaluées à travers ce qu'Ericson (2007, p. 380) appelle «les formats de communication» des systèmes de collecte de données:

Fixed-choice classification schemes, fill-in-the-blank forms, computerbased reporting systems and the like all embed regulations in the communication process. In doing so, they force organizational actors to simultaneously think, make decisions, act and report their actions in ways that appear procedurally correct and therefore accountable and legitimate.

Par conséquent, certaines résistances peuvent émerger à la suite de l'introduction de technologies dans les procès de travail policiers. Par exemple, des travaux ont souligné l'obstruction des policiers envers la fragmentation et le contrôle managérial accru de leurs tâches facilité par ces technologies. Meehan (1998) a en effet documenté les manières dont les policiers se les approprient au moyen de stratégies de défense de leur espace décisionnel.

Si elles formalisent la collecte et le traitement des données, les technologies compliquent aussi ces processus. Elles ajoutent aux pratiques de sécurité un coefficient de difficulté en exigeant des connaissances techniques poussées, un effet mis en lumière par les carences dans la formation des agents de première ligne en ce qui a trait à la manipulation des systèmes informationnels (Sanders \& Henderson, 2013). Ces technologies suscitent également de nouvelles interrogations quant aux interprétations faites par les travailleurs de la sécurité des données qu'il faut ou non colliger, et par extension, de la fiabilité de l'information inscrite dans ces systèmes. Elles multiplient les catégories et quantités de données à traiter et donnent lieu à des manquements dans leurs transmission et analyse selon les contextes de collectes, les ressources qui y sont affectées ainsi que le niveau de compatibilité des systèmes (Sheptycki, 2004; Ribaux \& Hicks, 2013). On peut donc, tout comme 
Bennett (2005) dans son examen de l'échange de données sur les passagers aériens, y aller d'une mise en garde envers les approches qui tiennent pour acquises l'homogénéité, l'efficience et l'interopérabilité des plateformes informatiques et des systèmes de renseignement aux frontières.

Les technologies ne font pas que réaménager la division du travail au sein des organisations de sécurité. Elles sont aussi différemment appréhendées par les acteurs qui en font usage et qui sont positionnés de façon inégale au sein du champ sécuritaire. Par exemple, tandis que l'usage des technologies de surveillance et de l'échange d'information par les agents sur le terrain peut contribuer au profilage racial et social (Norris \& Armstrong, 1999; Sanders \& Hannem, 2012), les technologies informationnelles sont conçues par les cadres intermédiaires, politiques et instances qui en font la promotion comme des outils favorisant une plus grande efficacité organisationnelle, l'interopérabilité dans le travail de sécurité ainsi que l'évaluation, voire la surveillance, du travail des professionnels de la sécurité. Les technologies s'inscrivent dans les registres de distinction propres aux milieux policiers (Proteau \& Pruvost, 2008). Tel que souligné par Brodeur et Dupont (2006), les tâches et les compétences sont valorisées de manière différentielle dans l'environnement policier et s'inscrivent dans les luttes d'influence propres à ce milieu. Bien que certaines formes de gestion des données peuvent être favorisées - pensons à l'analyse de renseignement -, d'autres comme la classification de documents ou la rédaction de rapports offrent peu de prestige aux professionnels de la sécurité.

Notre exploration de la mise en place d'une douane à distance grâce aux technologies de l'information et de surveillance des circulations s'appuie donc sur les débats contemporains qui émanent des études critiques sur la sécurité, des border studies et de la sociologie du policing. Parce qu'elle s'interroge sur les modalités du recours à ces technologies dans la conduite quotidienne des contrôles frontaliers et se questionne au sujet des fins pratiques à partir desquelles ces technologies sont déployées, notre étude jette une lumière inédite sur les enjeux de gouvernance des mobilités. Nous proposons maintenant d'étudier les usages concrets de ces technologies dans la régulation des flux de marchandises et ainsi, de remettre en question les relations sociotechniques qui rendent possible la diffusion des espaces frontaliers. Nous nous intéresserons ensuite aux enjeux de pouvoir sous-jacents à ces usages. 


\section{La douane à distance : nouvelles caractéristiques du travail douanier de première ligne}

Jusqu'à la moitié des années 1990, les douaniers couvraient l'ensemble du processus de mainlevée à la frontière. Ils recommandaient et exécutaient les décisions de renvoi d'un camion à l'inspection secondaire et décidaient quoi et comment inspecter. Ils classaient et administraient des quantités de formulaires douaniers et s'assuraient du respect des réglementations nationales (environnementales, sanitaires, produits dangereux, etc.). Ils offraient des services complets aux camionneurs, courtiers en douane, transporteurs et importateurs. Enfin, ils percevaient les taxes sur les importations.

Si les changements macro-économiques de cette décennie - avec, entre autres, la mise en œuvre de l'ALENA en 1994 - ont peu affecté les agents frontaliers responsables du contrôle des voyageurs dans les ports d'entrée terrestres, ils ont présenté des impacts significatifs sur le travail de traitement des camionneurs, des camions et des marchandises. Plusieurs des agents d'expérience se rappellent l'activité débordante créée dans les ports d'entrée par la montée rapide des échanges commerciaux transfrontaliers lors des années 1990. Ils se souviennent de files de camions longues de plusieurs kilomètres et de camionneurs patientant pendant plus d'une heure afin de remplir les formalités administratives alors d'usage à la frontière. Des pressions institutionnelles ont depuis requis des douaniers qu'ils se transforment en facilitateurs du commerce transfrontalier, un nouveau rôle particulièrement promu à travers l'introduction d'innovations technologiques qui visaient à éliminer les goulets d'étranglement à la frontière et qui ont transformé le travail de douane. Les premiers changements technologiques intégrés aux douanes canadiennes datent ainsi de la période qui précède les événements du 11 septembre 2001. Ces changements ne se présentent donc pas à cette époque comme une solution à des problèmes sécuritaires. Ils répondent plutôt à une tendance internationale de restructuration des services douaniers qui se retrouvent dès lors placés sous l'influence d'une logique néolibérale faisant la promotion d'une économie de marché mondialisée ainsi que de la circulation sans entraves des marchandises. 


\section{Déclarations électroniques}

Afin de répondre aux difficultés produites par l'augmentation rapide des flux de marchandises à la frontière, les services douaniers canadiens mettent en place, en 1997, le système de soutien de la mainlevée accélérée des expéditions commerciales (SSMAEC) - on appelle aussi EDI ces plateformes d'échange de données informatisées qui sont encore utilisées quotidiennement par les douaniers. Désormais, les importateurs et transporteurs peuvent transmettre électroniquement leurs données douanières préalablement à l'arrivée des marchandises aux ports d'entrée, ce qui permet une recommandation électronique pour la mainlevée du fret avant son arrivée à la frontière. Ce système a considérablement réduit la tâche administrative des douaniers et la manipulation de formulaires papier, ainsi que les temps d'attente à la frontière pour les camionneurs (d'environ une heure à moins de 30 secondes). Il a aussi minimisé le besoin pour les courtiers de douane d'être physiquement présents près des postes frontaliers à mesure que leurs clients adoptaient ce mode de transmission virtuel. Pendant notre terrain de recherche, nous avons pu constater la vacance des bureaux de plusieurs courtiers avoisinant les postes frontaliers, leurs opérations étant maintenant transférées dans des centres urbains. Grâce à d'autres initiatives adoptées à la suite d'EDI - telles que le programme d'autocotisation des douanes -, les grandes compagnies de transport et les importateurs majeurs approuvés par les services commerciaux de l'ASFC peuvent ensuite évaluer eux-mêmes leurs marchandises, transmettre leurs données et payer leurs taxes électroniquement.

Depuis, différentes bases de données internes ainsi que celles de la police fédérale (pensons au Centre d'information de la police canadienne, CPIC, administré par la Gendarmerie royale du Canada) ont été intégrées dans les postes frontaliers. Cependant, la principale modification dans l'organisation du travail introduite par la transmission électronique des données douanières consiste à étendre en amont le processus de prise de décision à la frontière. Les déclarations électroniques ont donc transformé les routines de travail des agents des services «commerciaux». Elles exigent des douaniers d'évaluer les expéditions à distance, c'est-à-dire sans pouvoir inspecter le conducteur du camion ou le fret au moment de la recommandation pour mainlevée. Ceux-ci peuvent désormais passer deux ou trois heures de leurs quarts 
de travail devant des écrans d'ordinateur à revoir ces déclarations et à les recommander pour la mainlevée (ou pour l'inspection secondaire).

Cette distanciation dans la pratique douanière se retrouve particulièrement dans certains ports d'entrée situés hors des centres urbains (par exemple des aéroports régionaux ou des petits postes éloignés). Dans ces postes, les agents évaluent électroniquement le fret qui entre au pays suivant d'autres modes de transport (aéroportuaire par exemple) et dont ils n'auront pas la possibilité d'examiner le contenu. En effet, suivant des mesures de réduction des coûts en 2008, EDI a permis la réorganisation de la division du travail non seulement au sein, mais aussi entre les ports d'entrée. Un agent qui relâche des cargaisons sur les «listes EDI» d'un aéroport situé à plusieurs centaines de kilomètres de son lieu de travail confie: "C'est ça qu'on trouvait difficile un peu au début, de faire ça à distance. On est habitué à l'avoir visuel, devant nous.» Cette réorganisation de la tâche entre postes frontaliers a eu pour effet de retirer la responsabilité de recommandation de la mainlevée aux agents de certains postes frontaliers afin de la redistribuer dans d'autres postes. Ce changement organisationnel a entraîné la suppression d'une partie importante des tâches des douaniers de ces postes où les heures passées à examiner les listes EDI sont maintenant dégagées.

\section{Ciblage et automatisation de la mainlevée}

Laccumulation de données sur les flux transfrontaliers de marchandises a favorisé le développement d'activités régionales centrées sur l'évaluation des risques ainsi que des tendances économiques et criminelles locales. À partir de ce moment, les évolutions technologiques commencent à associer des enjeux sécuritaires aux processus de facilitation des flux de marchandises. Deux fonctions correspondantes furent créées à cet effet dans les sections commerciales des postes frontaliers enregistrant les plus forts volumes de circulation. Ces deux fonctions convoitées offrent un pouvoir discrétionnaire significatif à ceux qui les occupent. Elles permettent l'exercice d'un large éventail de responsabilités relatives à l'analyse de données, des activités absentes du travail quotidien des agents de première ligne. La première position consiste à gérer le système de mainlevée automatisée pour les importateurs considérés «à faible risque» et présentant des antécédents de conformité avec la réglementation douanière. Selon le mois et le port, les agents 
rencontrés évaluent qu'entre 40 et $60 \%$ des entrées dans le SMAECK sont automatiquement recommandées à la mainlevée sans l'intervention d'un agent - qui conserve néanmoins l'autorité ultime de relâcher les marchandises et les camionneurs à la ligne d'inspection primaire. Le système comprend des contrôles aléatoires des exportateurs considérés conformes au niveau d'environ $2 \%$ des entrées, un pourcentage cité en entrevue et conforme aux standards internationaux en matière de contrôle des voyageurs et des expéditions.

Travaillant à partir d'analyses des tendances locales et de celles transmises par la section renseignement de l'ASFC, la deuxième fonction, le cibleur, permet d'inscrire dans le SSMAEC des «cibles» sur des produits spécifiques, des camionneurs, des entreprises de camionnage et des exportateurs afin que les douaniers procèdent à un questionnaire et à une inspection plus poussés. Essentiellement, le ciblage consiste en un travail de catégorisation des risques et des enjeux de conformité douanière. Les camions désignés par le cibleur ou par le système automatisé doivent être envoyés à l'inspection secondaire - soit sur une base aléatoire, soit parce que son chargement, son transporteur ou l'importateur a été ciblé pour inspection. Tandis que la relâche de marchandises représentait auparavant une tâche administrative laborieuse, le système automatisé et le ciblage limitent dorénavant la part prise par les douaniers dans cette activité. Par contre, il élargit le rôle des quelques agents responsables de ces systèmes; de collecteurs de données, ils deviennent analystes.

Manifeste électronique: intégration de la gestion des risques et de la relâche douanière

Ces deux postes locaux d'analyse de renseignements frontaliers sont maintenant remis en question par un programme qui intègre la relâche douanière et la gestion des risques. Le Manifeste électronique répond aux exigences de l'Organisation Mondiale des Douanes (OMD) tirées de l'initiative SAFE élaborée en 2005. L'OMD promeut auprès des pays membres l'utilisation de l'information commerciale préalable recueillie à travers «l'établissement de partenariats avec le secteur privé» à des fins d'évaluation des risques et de facilitation des échanges commer- 
ciaux $^{3}$. Au coût de plus de 400 millions de dollars et à la mise en œuvre maintes fois reportée, l'ASFC prévoit que le Manifeste électronique sera devenu opérationnel en $2014^{4}$. Contrairement à EDI qui fonctionnait sur une base volontaire, les participants sont maintenant tenus de présenter des informations détaillées quant au fret et au transporteur, mais également au camionneur, à l'importateur et à l'exportateur, et ce, au moins une heure avant le franchissement de la frontière.

La plateforme du Manifeste électronique peut opérer les diverses bases de données qui lui sont rattachées; une partie de l'évaluation des risques devient donc également automatisée ${ }^{5}$. Dans cette optique, l'ASFC expérimentait il y a peu avec la mise en place d'un «hub d'évaluation du risque commercial». Ce centre examine, analyse et cible les flux de transport routier au moyen de l'information fournie à travers le Manifeste électronique ${ }^{6}$. Basé à Windsor (le port d'entrée commercial le plus fréquenté à la frontière canado-étatsunienne) entre 2009 et 2011, ce projet pilote a mené au développement de neuf indicateurs de risque par lesquels les autorités frontalières peuvent procéder à un ciblage automatisé - ni ces indicateurs ni les critères d'évaluation utilisés dans

3. Organisation Mondiale des Douanes, 2005, L'OMD élabore un cadre de normes visant à sécuriser et à faciliter le commerce mondial, www.wcoomd.org/fr/media/ newsroom/2005/january/wco-develops-a-framework-of-standards-to-secure-andfacilitate-global-trade.aspx, (consulté le 15 octobre 2013).

4. ASFC, Initiative du Manifeste électronique - Étude d'évaluation. Rapport final août 2012, www.cbsa-asfc.gc.ca/agency-agence/reports-rapports/ae-ve/2012/emi-ime-fra. html (consulté le 15 octobre 2013).

5. Selon les positions (trafic, immigration, cargo aérien ou maritime, etc.) et cotes de sécurité, le e-Manifeste permet de consulter les bases de données suivantes: le Système de soutien de la mainlevée des expéditions commerciales (SSMAEC), le Numéro d'entreprise (NE), le Centre d'information de la police canadienne (CIPC), la Gestion des cotisations et des recettes de l'ASFC (GCRA), la Composante de recherche de marchandise (CRM), la Plateforme du commerce électronique des douanes (PCED), le Système de gestion de l'information des enquêtes des douanes (SGIEC), le Système de soutien des opérations des bureaux locaux (SSOBL) de Citoyenneté et immigration Canada, le Système de gestion de l'extraction de renseignements (SGER), le Système intégré d'exécution des douanes (SIED), le Système intégré des douanes (SID), la Ligne d'inspection primaire intégrée (LIPI), le Système de gestion des renseignements (SGR), le Système de récupération de renseignements judiciaires (SJJR), le Système d'information préalable sur les expéditions commerciales (TITAN) et le Système de traitement des déclarations des voyageurs (STDV). Cf.: ASFC, 2013, Source de renseignements du gouvernement fédéral et sur les fonctionnaires fédéraux 2013, www.cbsa-asfc.gc.ca/agency-agence/reports-rapports/pia-efvp/atipaiprp/infosource-fra.html\#a3x2 (consulté le 10 octobre 2013).

6. ASFC, Initiative du manifeste électronique - Étude d'évaluation, Rapport final août 2012, www.cbsa-asfc.gc.ca/agency-agence/reports-rapports/ae-ve/2012/emi-ime-fra. html (consulté le 15 octobre 2013). 
ces opérations ne sont divulgués publiquement. L’ASFC planifie l'établissement d'autres centres d'analyse dispersés dans quelques régions au pays - une information qui n'était pas encore rendue publique au moment de l'écriture de cet article. Le centre national de ciblage pour les modes aéroportuaire et maritime a quant à lui été inauguré à Ottawa en $2013^{7}$.

Puisque le ciblage commercial centralisé retravaille la manière et les motifs des inspections, il impose aux douaniers de nouvelles tâches de collecte d'informations détaillées destinées à être analysées par d'autres instances. En effet, avec le Manifeste électronique et la centralisation de la gestion des risques, les agents responsables des inspections secondaires sont tenus de recueillir des données spécifiques: la raison de l'inspection (aléatoire, renvoi par agent, cible), ce qui a été trouvé, la méthode de recherche (par exemple un échantillonnage aléatoire de produits ou une vérification approfondie), combien de boîtes ont été ouvertes, quelles sections du camion ont été inspectées, les marchandises examinées et leur pays d'origine. Il s'agit bien là de ces «formats de communication» dont Ericson (2007) a constaté la prédominance dans les milieux policiers. Non seulement une procédure d'exécution, les inspections se présentent désormais comme un travail d'accumulation de données. Elles s'inscrivent dans un réseau d'interventions de sécurité au sein duquel le port d'entrée n'apparaît plus que sous la forme d'un des nombreux points de contrôle investis par les opérations de gouvernance des flux de marchandises. En retour, afin d'évoluer et de développer des cibles précises et actualisées (par exemple les réseaux illicites de transport des drogues), ces activités de traçabilité des marchandises et de surveillance des travailleurs du transport apparaissent de plus en plus dépendantes de cette collecte d'information dans les ports d'entrée.

\section{«Ils placent cet aspect de plus en plus hors de notre portée » : déqualification et méfiance}

Comme c'est le cas pour la «police à distance» dans les espaces frontaliers, la mise en place d'une douane à distance repose sur l'intégration

7. ASFC, 2012, Vérification du Projet de mise en œuvre du programme national de ciblage, Rapport de vérification interne juin 2012, www.cbsa-asfc.gc.ca/agency-agence/ reports-rapports/ae-ve/2012/ntip-mpnc-fra.html (consulté le 9 octobre 2013). 
des ports d'entrée au sein d'un agencement de points de contrôle permettant de réguler les mobilités de marchandises. Néanmoins, notre recherche suggère que cette extension de l'espace frontalier est soutenue par ce que Ocqueteau et Dupont $(2013$, p. 8) désignent comme la «nouvelle division du travail [êtres humains]/machines de la mise en protection collective». Quels sont les effets concrets pour les douaniers de cette division du travail alors qu'elle se déploie dans l'espace frontalier technologisé? Nous avons vu que, historiquement, la classification et l'évaluation des voyageurs et des biens à partir d'inspections physiques ont été situées au cœur de l'organisation du travail douanier. Les activités douanières supposent depuis longtemps des pouvoirs discrétionnaires qui s'appuient sur des dispositifs légaux, une logique de suspicion et l'acquis expérientiel accumulé par les professionnels de la sécurité frontalière tout au long de leur carrière. Cependant, selon Lyon (2008, p. 36), on assisterait actuellement, dans des domaines tels que celui de la surveillance des mobilités de personnes, à un «déclin général des relations face-à-face» sous l'influence des technologies de communication. Or, ce sont ces relations qui rendent possible la mise en œuvre des pouvoirs discrétionnaires des agents de première ligne aux frontières.

Notre recherche confirme l'observation de Lyon non quant au nombre de ces relations mais quant à la diminution de leur portée. Le contrôle frontalier contemporain n'élimine pas les méthodes traditionnelles des douaniers - qu'il s'agisse de l'examen physique des cargaisons ou de l'évaluation de la conduite des voyageurs à partir de lectures discrétionnaires des comportements et autres «indicateurs de risque». Pourtant, sous l'effet du recours de plus en plus fréquent aux technologies et de la redistribution des tâches douanières et sécuritaires, le contrôle frontalier s'éloigne progressivement de ces méthodes et en marginalise l'importance dans le champ sécuritaire. Si les stratégies de gestion des risques limitent les possibilités offertes aux voyageurs de mettre en contexte leurs déplacements, elles réduisent aussi la marge d'action des douaniers. C'est ce que suggère Rygiel (2013, p. 153) lorsqu'elle affirme: «The reliance on expert techno-scientific knowledge subordinates other forms of border knowledge based on "everyday knowledge" [...] that accumulate over years as a border guard or that are the result of personal narrative and negotiation.» Ces modifications technologiques remettent en question le savoir pratique collectivement accumulé par les agents d'un poste frontière, à savoir leur connaissance 
des transporteurs locaux, des dynamiques économiques et criminelles régionales ainsi que la possibilité de questionner et d'examiner les camionneurs et les marchandises sur la base de ce savoir pratique.

Lorsque mise en œuvre dans le contexte frontalier, la gestion des risques permet donc davantage que la classification des choses et des personnes au moyen de «pratiques divisantes»- une notion proposée par Michel Foucault et maintes fois reprise dans les études sur les frontières, la sécurité et la surveillance. Elle affecte également la pratique professionnelle, les rôles et les responsabilités d'une diversité d'acteurs impliqués dans ces processus. Elle opère au sein des procès de travail en modifiant les tâches à accomplir et leur distribution. Elle génère de nouvelles hiérarchies au sein du champ sécuritaire non seulement entre fonctions, mais également entre lieux de contrôle - par exemple selon le volume des circulations traversant un poste frontalier particulier. La gouvernance technologique des circulations assure l'ascendant - ou bien le caractère marginal ou même le déclassement - d'un port d'entrée dans les réseaux de contrôle frontaliers en fonction de sa distance spatiotemporelle par rapport aux centres décisionnels et aux flux de marchandises dont ce port contribue à réguler la circulation.

Si l'on peut de plus en plus rendre compte des frontières comme construits sociotechniques (Frowd, 2014), les interactions technologiessécurité sont productrices de nouvelles tensions au sein des agences de sécurité. En effet, si l'introduction de technologies de l'information et de surveillance dans les opérations de sécurité transforme le rôle joué par les agents frontaliers dans la gouvernance des circulations, elle renouvelle également les enjeux de pouvoir dans lesquels s'inscrivent les douaniers. Si la diffusion de la frontière par des moyens technologiques tend à brouiller les responsabilités et à ébranler l'imputabilité déjà limitée des processus sécuritaires, c'est aussi parce qu'elle favorise une certaine déqualification du personnel frontalier de première ligne. À cet égard, la centralisation des tâches de ciblage et l'automatisation accrue de la relâche douanière entraînent une refonte du rôle des agents frontaliers de première ligne dont la contribution a été réduite dans la plupart des processus décisionnels. Dans ces conditions, il semble que les douaniers soient de plus en plus appelés à devenir de simples exécutants de références d'inspection générées aléatoirement ou selon des cibles précises, mais recommandées ailleurs dans l'espace frontalier.

On peut concevoir ici que la douane représente un cas d'école pour la gestion des mobilités dites à bas risque. En effet, certains programmes 
de dédouanement préétabli (par exemple, NEXUS en Amérique du Nord ou PARAFE dans l'Union européenne) annoncent l'adoption de schèmes de gouvernance de ces circulations qui visent non seulement à évaluer, mais également à faciliter les mobilités privilégiées de personnes telles que les gens d'affaires (Côté-Boucher, 2010). Cette facilitation peut bien sûr s'expliquer par les efforts faits par les autorités afin de maintenir la libre circulation des biens dans les espaces économiques continentaux développés au cours des années 1990. Mais il s’agit également de rappeler que ces modes de gestion des circulations sont maintenant préférés par les agences de sécurité alors qu'elles font face à des restrictions budgétaires qui exigent la concentration des ressources sécuritaires sur l'interdiction des mobilités dites à haut risque.

\section{Au sujet d'«Ottawa»: relations tendues entre agents de terrain et cadres intermédiaires}

Comme on peut s'y attendre, les douaniers témoignent de leur mécontentement relativement à leur mise à l'écart croissante des processus décisionnels frontaliers. Ils accueillent avec ambivalence les changements technologiques et organisationnels décrits plus haut. Un douanier explique sa réserve:

Les autres agents qui contrôlent les entrées de notre poste ne savent pas ce qu'ils cherchent. Ce serait comme si j'essayais de cibler un port maritime. Je n'ai jamais travaillé dans un port. Essayer de cibler ça pour moi... Je n'ai pas une compréhension réelle de leurs opérations. [...] Ce n'est pas la meilleure façon de procéder, n'est-ce pas? Ils [1'Agence] placent cet aspect de plus en plus hors de notre portée.

S'il est difficile d'obtenir de l'information concernant les méthodes des nouvelles unités de ciblage de l'ASFC, les participants à notre recherche qui connaissaient ces projets ont exprimé avec franchise leurs réserves. Ils craignaient que cette initiative ne supprime les activités locales de ciblage et de mainlevée automatisée. Un autre agent explique:

Jusqu'à ce que le débat entre les régions et Ottawa sur le niveau du ciblage soit réglé... Pour l'instant, il semble que c'est Ottawa qui ait gain de cause. À Windsor d'ailleurs, ils ont ouvert le centre de ciblage national. Donc, on s'oppose à du ciblage régional ou local [...]. Les régions se battent pour avoir une forme de ciblage, ou maintenir ce qu'elles ont. Donc, les phénomènes locaux, c'est sûr que Windsor ne pourra pas bien les représenter. Les 
agents ici vont devoir insérer probablement les phénomènes locaux qui arrivent et ça va être transmis à Windsor. C'est Windsor qui va le faire.

Une troisième agente ajoute:

C'est le même problème qu'on va avoir: toute la vérification des marchandises et des importations va être faite avant et c'est les gens qui sont à Windsor qui vont décider de les envoyer à notre dock pour faire un examen. [...] Donc si la gestion ne pense pas qu'il va y avoir plus d'examens - parce que c'est eux qui vont gérer ça, le nombre d'examens qu'ils nous envoient -, s'ils ne transfèrent pas leur personnel, on va se trouver avec beaucoup d'examens et puis personne pour les faire. C'est le même style que la mainlevée automatisée. [...]

C'est dommage parce que nous, on connaît notre clientèle. On connaît ce qui passe ici, on connaît la marchandise. Tandis que là-bas, ils ne connaissent pas notre clientèle, ils vont cibler à peu près n'importe quoi, et c'est eux qui vont décider pour nous ce qu'on doit vérifier.

Les douaniers se sont révélés critiques quant aux interactions sociotechniques qui retirent la décision douanière de son ancrage local. Les commentaires des participants à cette recherche quant à la centralisation du ciblage remettent en question le présupposé d'une sécurité accrue qui serait obtenue grâce au recours aux technologies de l'information et de la surveillance. Ces douaniers soulignent comment les nouveaux enjeux criminels et de sécurité régionaux peuvent échapper à des services de renseignement frontaliers situés à la capitale. La connaissance limitée des dynamiques locales par ces derniers pourrait en fait entraver la mise en place d'actions de sécurité réellement ciblées. De plus, les douaniers s'interrogent sur la pertinence de certaines recommandations de ces services d'analyse centralisés qui exigent des inspections que les agents de terrain considèrent inutiles - soit parce que la compagnie de transport ou le camionneur ciblés sont connus des services frontaliers locaux et ont déjà fait l'objet de plusieurs examens, soit parce que, faute d'espace disponible sur les docks, ces inspections obligatoires préviennent la tenue d'autres inspections décidées par les douaniers euxmêmes.

Nos entretiens révèlent une autre dimension de cet état d'insatisfaction par rapport aux décisions prises par l'administration centrale et invitent à élaborer une lecture sociologique de l'impact des technologies sur les organisations de sécurité. Dans leur méfiance affichée envers les gestionnaires de la capitale se lit l'impuissance ressentie par les doua- 
niers à l'égard de mécanismes de distanciation qui transforment leur travail et marginalisent leur apport à la prise de décision. Les agents rencontrés ont exprimé leur frustration répétée de ne pas être suffisamment consultés au sujet de nouvelles procédures douanières. Ils affirment ne pas être convenablement formés pour mettre en œuvre des programmes imaginés ailleurs et se considèrent cobayes de systèmes créés par les services informatiques fédéraux. Ce sens d'être peu à peu mis à l'écart dans la prise de décision frontalière s'est souvent exprimé en entrevue dans la méfiance des agents envers les gestionnaires de la capitale, mais aussi dans le sentiment que leur travail n'est pas respecté par «Ottawa». Les décideurs ne posséderaient pas le savoir-faire nécessaire: «Ottawa, ils ne connaissent pas les opérations.» «Ottawa, ils ne savent même pas ce que c'est que la douane.» Un vocable désignant aussi bien les politiciens que la haute direction des autorités frontalières, les mentions d'«Ottawa» dépeignent les autorités frontalières de la capitale comme étant déconnectées du terrain ${ }^{8}$. Faire allusion à "Ottawa», c'est aussi renvoyer à une conception du siège social de l'ASFC comme celle d'un centre de pouvoir inaccessible pour la plupart des agents travaillant en région. Cette position symbolique de la capitale a été illustrée par de fréquentes références à sa situation hiérarchique élevée à travers des expressions telles que «up there», «la haute direction» et «the powers that be».

Bien sûr, il n'est pas rare pour des fonctionnaires de première ligne et les cadres intermédiaires d'une bureaucratie d'être en désaccord sur le mandat de leur organisation, sur les méthodes de travail à utiliser et sur les objectifs à poursuivre (Lipsky, 2010 [1980]). Cependant, la réorganisation des procès de travail par leur technologisation et la diminution progressive des responsabilités d'application des lois et réglementations à partir des postes frontaliers constituent des processus qui retravaillent les luttes d'influence au sein de ces agences. Ainsi, le recours aux technologies exacerbe les tensions existantes entre les

8. Nous avons rencontré trois agents qui, par le passé, avaient obtenu des contrats à «Ottawa» afin d'y travailler sur des projets de l'ASFC et d'autres qui envisageaient d'y déménager afin d'y poursuivre leur carrière dans la fonction publique fédérale. Malgré ces efforts institutionnels visant à favoriser le transfert des savoirs pratiques des régions aux quartiers généraux, ces agents ont témoigné du peu de connaissance du terrain affiché par leurs collègues de la capitale. Alimentant les conflits intraorganisationnels, ces histoires au sujet d'«Ottawa» circulent au sein des postes frontaliers dès que les agents terminent ces contrats ou lors de contacts informels visant à l'échange d'information et de bons procédés entre ces agents en poste à la capitale et leurs anciens collègues travaillant en port frontalier. 
gestionnaires basés dans la capitale et les agents travaillant sur le terrain. Les récentes enquêtes sur les employés fédéraux soulignent un des effets de ces luttes sur les conditions de travail; les agents de l'ASFC se retrouvent à l'échelon le plus bas de satisfaction au travail dans l'appareil d'État, rivalisant seulement avec les employés des services correctionnels ${ }^{9}$. Il faut également souligner que ces tensions internes suscitées par les réformes des tâches des douaniers se répètent à l'international. Ce fut le cas avec la grève des agents frontaliers britanniques de la UKBA en 2011 à la suite de débats autour d'une réduction des contrôles sécuritaires en raison de l'engorgement de certains ports d'entrée britanniques. Nos conclusions font également écho à celles de Brenda Chalfin (2010) qui, dans son ethnographie de l'impact des mesures néolibérales sur les douanes ghanéennes, a constaté que la perte d'autorité des agents de première ligne consécutive à la centralisation des pouvoirs frontaliers avait amplifié les tensions entre les douaniers et les dirigeants des services frontaliers. Ces luttes d'influence nous permettent donc de remettre en question le discours d'efficience et de neutralité invariablement adopté par ceux qui font l'apologie de l'automatisation des processus de sécurité. Les technologies de l'information renouvellent plutôt les enjeux de pouvoir au sein des organisations de sécurité.

\section{Conclusion}

Cet article a prêté une attention particulière à la manière dont les technologies de contrôle frontalier altèrent les routines de travail des douaniers. Alors que ce que nous avons appelé ici la douane à distance permet d'accélérer la prise de décision à la frontière, il faut admettre que les frontières contemporaines n'apparaissent pas seulement comme le résultat d'une surveillance diffuse des mobilités facilitée par les technologies de l'information. En effet, les dynamiques qui influent sur la conduite de la sécurité frontalière résultent également du travail concret réalisé par des professionnels de la sécurité. Ces dynamiques répondent en partie de leurs efforts vers la préservation de leur influence à la suite des changements organisationnels et technologiques qui

9. Secrétariat du Conseil du Trésor du Canada, Sondage auprès des fonctionnaires fédéraux 2011, www.tbs-sct.gc.ca/pses-saff/2011/introduction-fra.asp (consulté le 19 mai 2014). 
travaillent le champ sécuritaire de l'intérieur. Depuis l'introduction de technologies de gestion de risque et de facilitation douanière, certaines des tâches qui occupaient les douaniers pendant une grande partie de leurs quarts de travail ont été redistribuées dans le champ sécuritaire, réduites ou tout simplement éliminées. Par conséquent, les processus de distanciation contemporains qui influent sur le contrôle des frontières s'accompagnent d'une déqualification partielle des douaniers. Cette refonte de la gouvernance des mobilités creuse donc les tensions existantes au sein de l'autorité frontalière entre ces agents de première ligne opérant en région et les gestionnaires basés à la capitale. Les réflexions des douaniers quant à leur capacité de mener à bien leurs tâches quotidiennes et au sujet de leur mise à l'écart progressive des processus décisionnels nous permettent de mieux cerner les effets concrets des mutations politiques, technologiques et organisationnelles contemporaines en matière de sécurité frontalière.

\section{Références}

Aas, K. F. (2006). 'The body does not lie': Identity, risk and trust in technoculture. Crime, Media, Culture, 2(2), 143-158.

Aas, K. F. (2011). 'Crimmigrant' bodies and bona fide travelers: Surveillance, citizenship and global governance. Theoretical Criminology, 15(3), 331-346.

Amoore, L. (2006). Biometric borders: Governing mobilities in the war on terror. Political Geography 25(3), 336-351.

Amoore, L., \& de Goede, M. (Éds.). (2008). Risk and the War on Terror. London: Routledge.

Balibar, E. (2009). Europe as borderland. Environment and Planning D: Society and Space, 27(2), 190-215.

Bennett, C. J. (2005). What happens when you book an airline ticket? The collection and processing of passenger data post 9/11. Dans E. Zureik \& M. B. Salter (Éds.), Global Surveillance and Policing (pp. 113-138). Portland, OR: Wilan.

Bigo, D., \& Guild, E. (2005). Policing at a distance: Schengen visa policies. Dans D. Bigo \& E. Guild (Éds.), Controlling Frontiers: Free Movement Into And Within Europe (pp. 233-263). Aldershot: Ashgate.

Bonditti, P. (2005). Biométrie et maîtrise des flux: vers une «géo-technopolis du vivant-en-mobilité». Cultures $\mathbb{W}$ Conflits, 58, 1-16.

Brodeur, J.-P., \& Dupont, B. (2006). Knowledge Workers or "Knowledge" Workers? Policing \& Society, 16(1), 7-26.

Broeders, D. (2007). The New Digital Borders of Europe: EU Databases and the Surveillance of Irregular Migrants. International Sociology, 22(1), 71-92. 
Ceyhan, A. (2008). Technologization of security: Management of uncertainty and risk in the age of biometrics. Surveillance and Society, 5(2), 102-123.

Chalfin, B. (2007). Customs regimes and the materiality of global mobility: Governing the port of Rotterdam. American Behavioral Scientist, 50(12), 16101630.

Chalfin, B. (2010). Neoliberal Frontiers. An Ethnograpby of Sovereignty in West Africa. Chicago, IL: Chicago University Press.

Chan, J. (2001). The technological game: How information technology is transforming police practice. Criminal Justice, 1(2), 139-159.

Chan, J., Brereton, D., Legosz, M., \& Doran, S. (2001). E-policing: The Impact of Information Technology on Police Practice. Queensland Criminal Justice Commission.

Côté-Boucher, K. (2008). The diffuse border: intelligence-sharing, control and confinement along Canada's Smart Border. Surveillance and Society, 5(2), 142-165.

Côté-Boucher, K. (2010) Risky business? Border preclearance and the securing of economic life in North America. Dans S. Braedley \& M. Luxton (Éds.), Neoliberalism and Everyday Life (pp. 37-67). Montreal: McGill-Queen's.

Côté-Boucher, K., Infantino, F., \& Salter, M. (2014). Border security as practice. An agenda for research. Security Dialogue, 45(3), 195-208.

Doty, R. (2007). States of exception on the Mexico-U.S. border: Security, "decisions", and civilian border patrols. International Political Sociology, 1(2), 113-137.

Ericson, R. V. (2007). Rules in policing: Five perspectives. Theoretical Crimino$\log y, 11(3), 367-401$.

Ericson, R. V., \& Haggerty, K. D. (1997). Policing the Risk Society. Toronto: University of Toronto Press.

Frowd, P. M. (2014). The field of border control in Mauritania. Security Dialogue, 45(3). doi: 10.1177/0967010614525001.

Gilboy, J. (1991). Deciding who gets in: decisionmaking by immigration inspectors. Law $\mathfrak{F}$ Society Review, 25(3), 571-600.

Heyman, J. McC. (1995). Putting power in the anthropology of bureaucracy. The immigration and naturalization service at the Mexico-United States border. Current Anthropology, 4(1), 261-287.

Heyman J. McC. (2004) Ports of entry as nodes in the world system. Identities: Global Studies in Culture and Power 11(3): 303-327.

Huysmans, J. (2008). The jargon of exception-On Schmitt, Agamben and the absence of political society. International Political Sociology, 2(2), 165-183.

Infantino, F., \& Rea, A. (2012). La mobilisation d'un savoir pratique local: attribution des visas Schengen au consulat général de Belgique à Casablanca. Sociologies pratiques, 24, 67-78.

Jeandesboz, J. (2010). Logiques et pratiques de contrôle et de surveillance des frontières de l'Union européenne. Dans A. Scherrer, E.-P. Guittet \& D. Bigo 
(Éds.), Mobilité(s) sous surveillance. Perspectives croisées UE-Canada (pp. 129147). Montréal: Athéna éditions.

Lipsky, M. (2010 [1980]). Street-Level Bureaucracy: Dilemmas of the Individual in Public Services (30th anniversary expanded edition). New York, NY: Russell Sage Foundation.

Lyon, D. (2007). Surveillance, security and social sorting. Emerging research priorities. International Criminal Justice Review, 17(3), 161-170.

Lyon, D. (2008). Filtering flows, friends, and foes: Global surveillance. Dans M. B. Salter (Éd.), Politics at the Airport (pp. 29-50). Minneapolis, MN: University of Minnesota.

Meehan, A. J. (1998). The impact of mobile data terminal (MDT) information technology on communication and recordkeeping in patrol work. Qualitative sociology, 21(3), 225-254.

Norris, C., \& Armstrong, G. (1999). The Maximum Surveillance Society: The Rise of CCTV. Oxford: Berg.

Ocqueteau, F., \& Dupont, B., (2013), Introduction. Nouveaux regards sur les métiers de sécurité. Criminologie, 46(2), 5-13.

Pickering, S., \& Weber, L. (Éds.). (2006). Borders, Mobility and Technologies of Control. Dordrecht: Springer.

Pratt, A., \& Thompson, S. (2008). Chivalry, 'race' and discretion at the Canadian border. The British Journal of Criminology, 48(5), 620-640.

Pratt, A. (2010). Between a hunch and a hard place: Making suspicion reasonable at the Canadian border. Social $\leftarrow$ Legal Studies, 19(4), 461-480.

Proteau, L., \& Pruvost, G. (2008). Se distinguer dans les métiers d'ordre (armée, police, prison, sécurité privé). Sociétés contemporaines, 72(déc.), 7-14.

Ribaux, O., \& Hicks, T. (2013). Technology and database expansion: what impact on policing? Dans S. Leman-Langlois (Éd.), Technocrime, Policing and Surveillance (pp. 91-109). Abingdon: Routledge.

Rygiel, K. (2010). Globalizing Citizenship. Vancouver: UBC Press.

Rygiel, K. (2013). Mobile citizens, risky subjects: security knowledge at the border. Dans S. Ilcan (Éd.), Mobilities, Knowledge, and Social Justice (pp. 152176). Montreal/Kingston: McGill-Queen's University Press.

Salter, M. (2007). Governmentalities of an Airport: Heterotopia and Confession. International Political Sociology, 1(1), 49-66.

Salter, M. (2008). When exception becomes the rule: borders, sovereignty, and citizenship. Citizenship Studies, 12(4), 365-380.

Sanders, C. B., \& Hannem, S. (2012). Policing "the risky": Technology and surveillance in everyday patrol work. Canadian Review of Sociology/Revue canadienne de sociologie, 49(4), 389-410.

Sanders, C. B., \& Henderson, S. (2013). Police 'empires' and information technologies: uncovering material and organisational barriers to information sharing in Canadian police service. Policing and Society: An International Journal of Research and Policy, 23(2), 243-260. 
Scherrer, A. (2013). Lutte antiterroriste et surveillance du mouvement des personnes. Criminologie, 46(1), 15-31.

Scherrer A, Guittet E.-P., \& Bigo, D. (dir), (2010). Mobilités sous surveillance. Perspectives croisées UE-Canada. Montréal: Athéna Éditions.

Sheptycki, J. (2004). Organizational pathologies in police intelligence-systems: Some contributions to the lexicon of intelligence-led policing. European Journal of Criminology, 1(3), 307-332.

Squire, V. (Éd.). (2011). The Contested Politics of Mobility: Borderzones and irregularity. London: Routledge.

Torpey, J. (2000). The Invention of the Passport. Surveillance, citizenship and the state. Cambridge: Cambridge University Press.

Walters, W. (2006). Border/Control. European Journal of Social Theory, 9(2), 187-203.

Weber, L., \& Bowling, B. (2004). Policing Migration: A Framework for Investigating the Regulation of Global Mobility. Policing and Society: An International Journal of Research and Policy, 14(3), 195-212.

Wilson, D., \& Weber, L. (2008). Surveillance, risk and preemption on the Australian border. Surveillance and Society, 5(2), 121-141.

ABSTRACT - Border and security studies have established that technologies are at the centre of the reconstruction of border spaces and border policing activities. The aim of this article is to explore the little studied domain of customs, that is, where border authorities are concerned with the traceability of goods and the movement of transport workers. Drawing on field research conducted in the "commercial" sections of five Canadian border crossings, we studied the concrete effects produced by the integration of surveillance and information technologies in the customs labour process. The article underscores how customs officers establish themselves as security actors, but also pays attention to the daily practices of border security professionals, as well as labour conditions and intra-organizational relations within border security agencies. Instead of increased efficiency, which the use of technologies ought to provide, this integration results in struggles between practitioners and middle managers due to deskilling and the automation of customs work.

KEYWORDS • Border security, customs, technologies, deskilling, power struggles.

RESUMEN - Los trabajos sobre la seguridad en las fronteras han establecido claramente que las tecnologías se sitúan en el corazón de la reconstitución de las actividades de la policía aduanera y del espacio ocupado por las fronteras. El presente artículo tiene como objetivo de abrir perspectivas hacia el mundo poco estudiado de las aduanas, es decir, alli donde se preocupan del rastreo de las mercaderías y de los desplazamientos de los trabajadores del transporte. Basándonos en una investigación de terreno llevada a cabo en las divisiones "comerciales" de cinco puestos fronterizos canadienses, nos preguntamos sobre los efectos concretos de la integración de las tecnologías en el proceso laboral de los aduaneros. Nos inclinamos a la manera en la que los aduaneros se constituyen como actores de seguridad, pero, igualmente, a las prácticas cotidianas de los profesionales de la seguridad de las fronteras, a las condiciones de trabajo y a las relaciones intra-institucionales en el seno de dichas agencias de seguridad fronte- 
riza. En efecto, más que una mayor eficiencia, que sería obtenida mediante el recurso a las tecnologías, dicha integración presenta escollos que revelan, por lo bajo, luchas de influencia entre los actores del terreno y los superiores intermedios, junto, como trama de fondo, la descalificación y la automatización del trabajo aduanero.

PALABRAS CLAVE - Seguridad fronteriza, aduanas, tecnologías, descalificación, luchas de influencia. 